\title{
Evolution of Diabetic Maculopathy from Marked Exudation to Subretinal Fibrosis: Clinical and Spectral Domain Optical Coherence Tomography Features
}

\author{
Daniel S. Churgin, Jonathan H. Tzu, Harry W. Flynn \\ Department of Ophthalmology, Bascom Palmer Eye Institute, University of Miami Miller School of Medicine, \\ Miami, FL, USA \\ Email:HFlynn@med.miami.edu
}

Received 20 August 2015; accepted 13 November 2015; published 16 November 2015

Copyright (C) 2015 by authors and Scientific Research Publishing Inc.

This work is licensed under the Creative Commons Attribution International License (CC BY). http://creativecommons.org/licenses/by/4.0/

\section{(c) (i) Open Access}

\begin{abstract}
Diabetic maculopathy with marked exudation may lead to subretinal fibrosis. Two patients observed over multiple years evolved from macular exudation into subretinal fibrosis with severe visual loss. Spectral domain optical coherence tomography and color photographs document the clinical changes.
\end{abstract}

Keywords

Diabetes Mellitus, Retinopathy, Subretinal Fibrosis, Exudate, OCT, Maculopathy

\section{Introduction}

Marked exudation in the macula center of patients with advanced diabetic retinopathy is associated with significant visual loss, which may not improve with currently available treatments [1]-[7]. This case series documented spectral domain optical coherence tomography (SD-OCT) diagnosis and clinical outcomes of two patients with diabetes-related severe macular exudation, which evolved into subretinal fibrosis.

\section{Case Series}

\subsection{Case 1}

A 66-year-old type II diabetic woman presented with a history of decreased vision of the left eye for 3 years.

How to cite this paper: Churgin, D.S., Tzu, J.H. and Flynn, H.W. (2015) Evolution of Diabetic Maculopathy from Marked Exudation to Subretinal Fibrosis: Clinical and Spectral Domain Optical Coherence Tomography Features. Open Journal of Ophthalmology, 5, 158-162. http://dx.doi.org/10.4236/ojoph.2015.54025 
The patient had previously received focal photocoagulation in the left eye. Initial best-corrected visual acuity (BCVA) was 20/40 in the right eye and 20/400 in the left eye. Examination showed advanced non-proliferative diabetic retinopathy in both eyes, and marked macular exudation in the left eye with foveal involvement (Figure 1(a)). SD-OCT showed distorted foveal contour, intraretinal fluid, and subretinal exudate (Figures 1(b)-(d)). The patient underwent multiple intravitreal injections of bevacizumab for macular edema in the left eye, which eventually resolved. However, the large area of macular exudation in the left eye contracted over the following two years, and evolved into subretinal fibrosis (Figure 2(a)). Repeat SD-OCT showed distorted foveal contour, trace intraretinal fluid, and subretinal fibrosis (Figures 2(b)-(d)).Visual acuity remained 20/200 in the left eye over four years.

\subsection{Case 2}

A 59-year-old patient with type II diabetes presented with a history of decreased vision of the right eye for 5 years. The patient had not previously received treatment for diabetic retinopathy. Initial best-corrected visual acuity (BCVA) was 5/200 E in the right eye and 20/100 in the left eye. Examination showed advanced non-proliferative diabetic retinopathy in both eyes, and marked macular exudation and edema in the right eye with foveal involvement (Figure 3(a)), and moderate macular exudation and edema in the left eye with foveal involvement. SD-OCT of the right eye showed distorted foveal contour, retinal atrophy, intraretinal fluid, and very early subretinal exudate (Figures 3(b)-(d)). The patient underwent multiple intravitreal injections of bevacizumab, aflibercept, and triamcinolone acetonide for macular edema of both eyes. The area of macular and foveal exudation of the right eye worsened over the following three years, and the patient developed subretinal fibrosis (Figure 4(a)). Repeat SD-OCT of the right eye showed thinned and distorted foveal contour, intraretinal fluid, and subretinal fibrosis (Figures 4(b)-(d)). Visual acuity remained 5/200 E in the right eye over two years, and appearance on fundus examination and OCT remained stable.

\section{Discussion}

Although infrequently described in the literature, diabetes-related macular exudates may evolve into subretinal

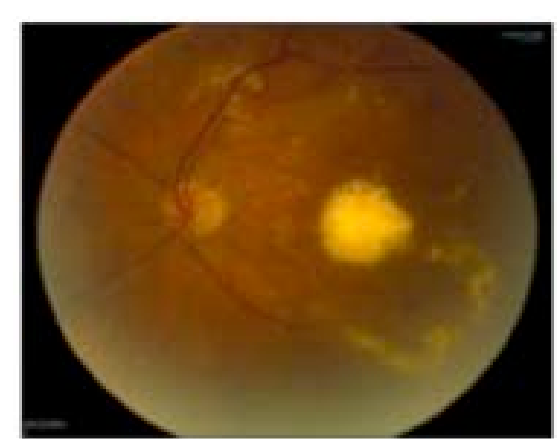

(a)

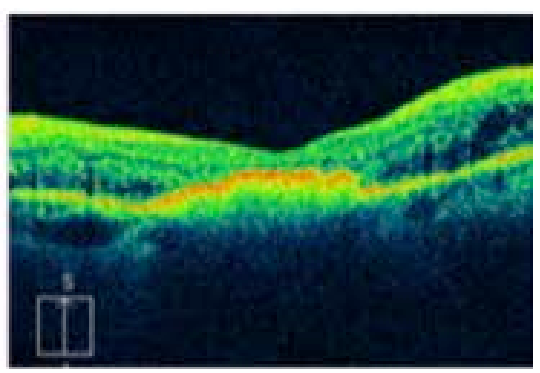

(c)

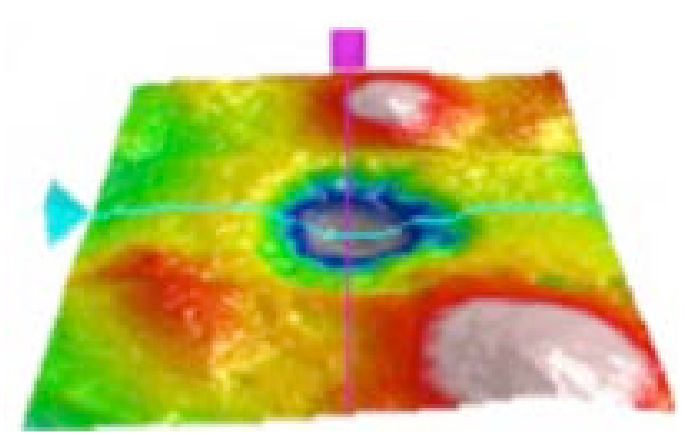

(b)

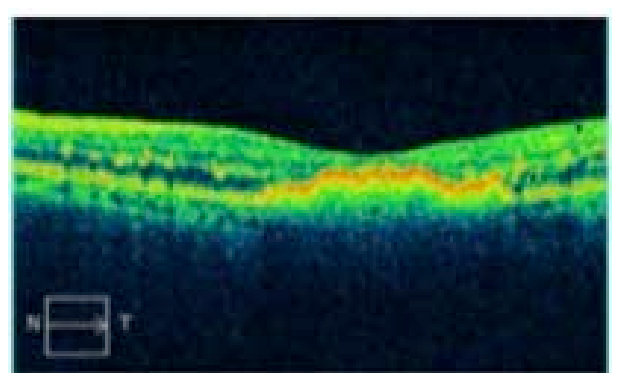

(d)

Figure 1. (a) At presentation, color image shows severe exudation with foveal involvement in the left eye. (b) SD-OCT topography map shows circinate rings of edema surrounding the macular center, which has relative thinning. (c) (d) SD-OCT demonstrates abnormal foveal contour, intraretinal fluid, and subfoveal exudation. 


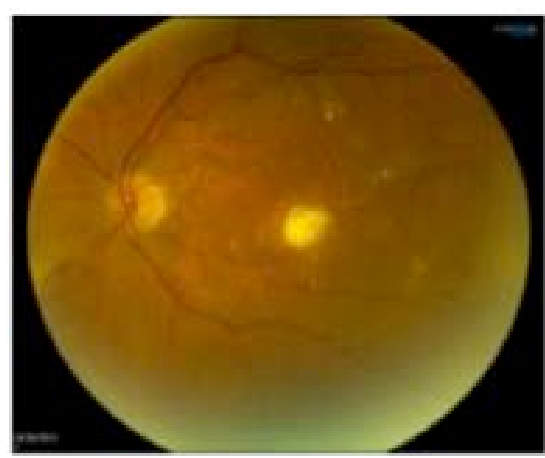

(a)

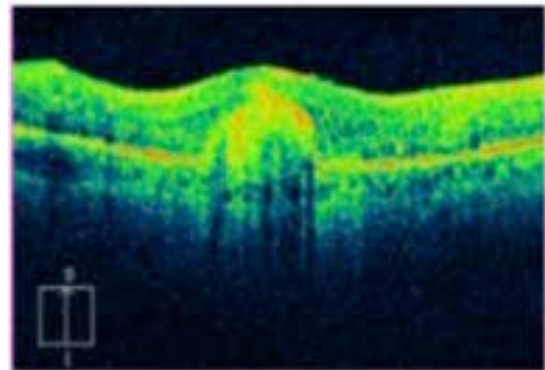

(c)

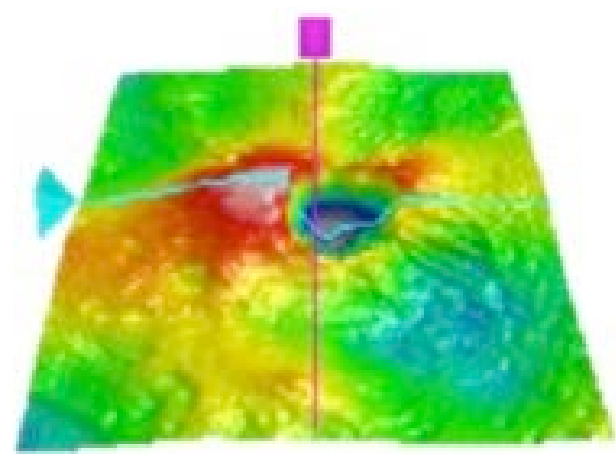

(b)

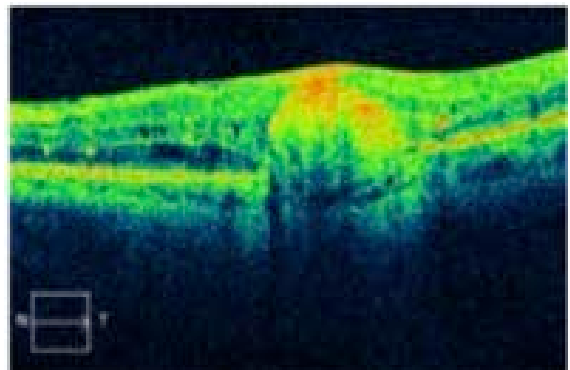

(d)

Figure 2. (a) Four years after initial presentation, color image shows contracted white subretinal fibrotic lesion. (b) Topography map shows persistent edema surrounding the macula center. (c) (d) SD-OCT shows persistent abnormal foveal contour, intraretinal fluid, and subretinal fibrosis involving the fovea.

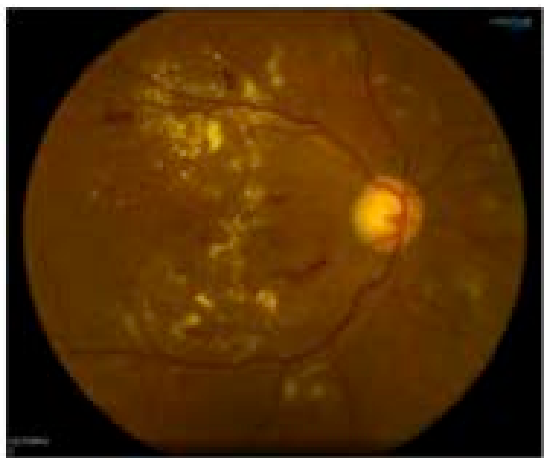

(a)

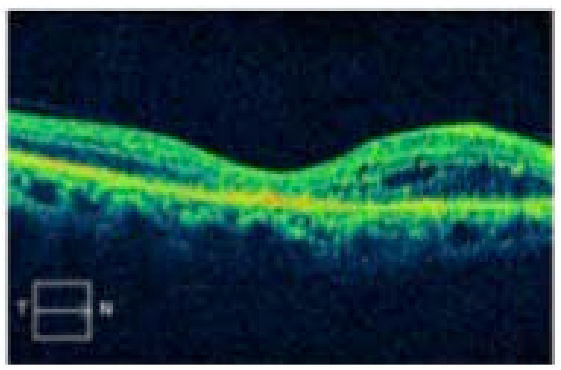

(c)

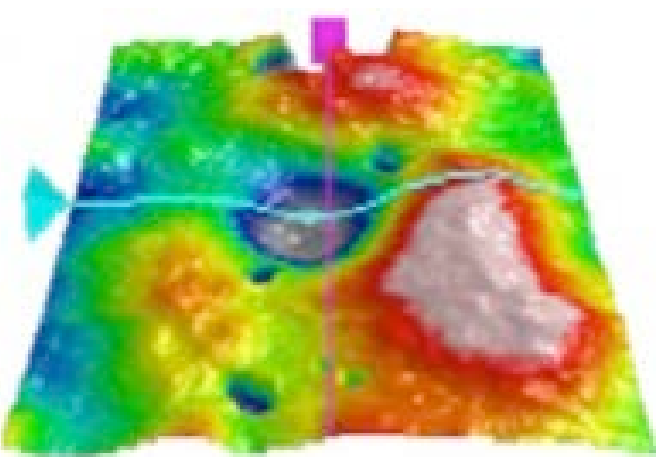

(b)

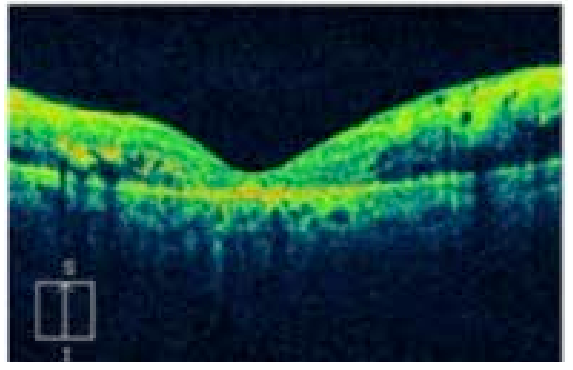

(d)

Figure 3. (a) At patient presentation, color image shows marked exudation with foveal involvement. (b) The topography map shows a large circinate ring of edema nasal to the fovea. (c) (d) SD-OCT demonstrates subfoveal exudate, intraretinal fluid, and relative thinning of the fovea. 


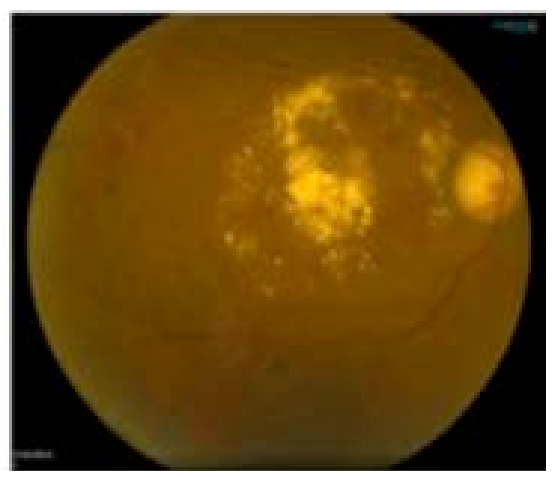

(a)

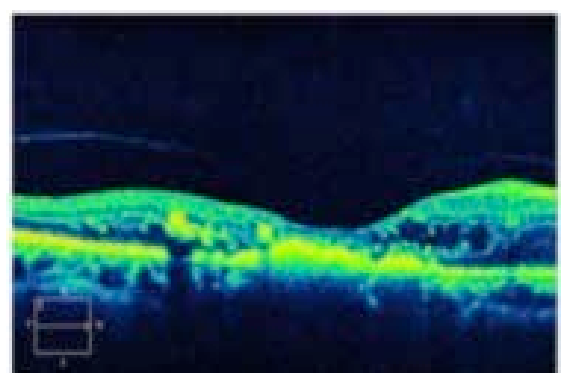

(c)

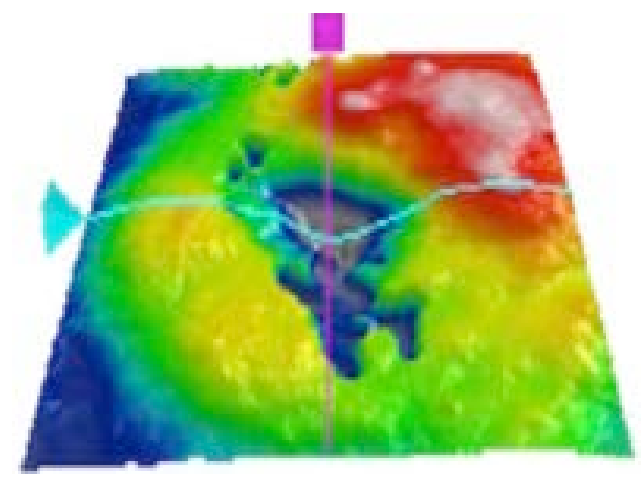

(b)

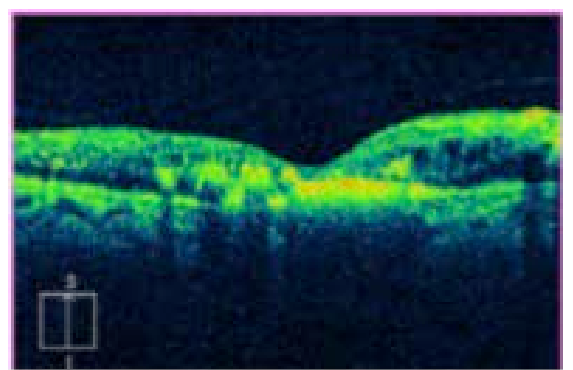

(d)

Figure 4. (a) Three years after initial presentation, color image shows worsened macular exudates. (b) Topography map shows persistent edema nasal to the fovea. (c) (d) SD-OCT shows foveal thinning, intraretinal fluid, and an organized plaque of subretinal fibrosis.

fibrosis. Clinicopathologic study of an organized plaque in exudative diabetic maculopathy was reported in 1976. In this case report, a poorly controlled diabetic patient was observed over the final years of the patient's life, during which time prominent macular exudates evolved into subretinal fibrosis. At autopsy, the authors observed diabetic microangiopathy, retinal degeneration and proliferation, and serous detachment of the pigment epithelium. The underlying Bruch's membrane and choriocapillaris were unaffected, suggesting that the origin of the subretinal fibrosis was from the retinal exudate. They theorized that cystoid macular edema and hard exudates disrupted the retina and later caused atrophy and degeneration, along with proliferation and serous separation of the pigment epithelium. The authors proposed that this space could then be filled in by fibrous tissue from both retinal and RPE origins [2]. In a later case series, the same authors further proposed that this large accumulation of exudates may incite metaplasia and over time, with resultant organization into a fibrotic scar [3]. In these five patients observed in these cases, vision was poor at presentation, and remained poor over long-term follow up [2] [3]. However, visual outcome for subretinal fibrosis may improve if the fovea is spared [4].

The largest case series of patients with subretinal fibrosis in the setting of diabetes was reported in the Early Treatment Diabetic Retinopathy Study (ETDRS) Report 23. The authors observed 5633 eyes with clinically significant macular edema (CSME) and no fibrotic changes, in contrast to 109 eyes with CSME and subretinal fibrosis. Of these 109 eyes with fibrosis, 74\% of the eyes had very severe hard exudates in the macula before developing subretinal scarring [1]. Subretinal fibrosis has been described in association with higher burn intensity during focal photocoagulation [5]-[8], and the ETDRS authors also sought to discover whether focal photocoagulation was the root cause of subretinal fibrosis in diabetics. They observed that only 9 of the 109 eyes had focal photocoagulation adjacent to the subretinal fibrosis. In this study of 264 eyes with very severe hard exudates, $30.7 \%$ of these eyes showed the presence of subretinal fibrosis. The ETDRS study group concluded that although focal photocoagulation might be a risk factor for development of subretinal fibrosis, the strongest risk factor for development of subretinal fibrosis was the presence of very severe hard exudates in the macula. One important limitation of this study is that the study occurred prior to the use of OCT. Therefore, the exact location of the exudates could not be determined [1].

The patients in this case series add evidence to the body of literature that very severe diabetic macular exuda- 
tion can evolve into subretinal fibrosis. In agreement with the ETDRS authors, it seems that the fibrosis develops in association with the severe exudation, as opposed to nearby focal laser scars. Vision remained poor but stable in these patients, and the disease followed a predictable course, evolving over time into subretinal fibrosis. In this era of anti-vascular endothelial growth factor widespread availability, both patients underwent multiple intravitreal injections of anti-vascular endothelial growth factor agents in order to treat CSME. There was no alteration in visual acuity or change in the natural course of this disease with these agents. Surgical removal of large foveal hard exudates has been reported, but visual improvement was minimal [9]. One small case series described the improvement of less severe exudates with the use of intravitreal triamcinolone [10]. A larger prospective study using SD-OCT would be needed to evaluate the course of prominent macular exudates undergoing frequent anti-VEGF or steroid injections. In addition, improved blood glucose control, blood pressure optimization, and control of serum lipids may improve the clinical course in these patients.

\section{References}

[1] Fong, D.S., Segal, P.P., Myers, F., Ferris, F.L., Hubbard, L.D. and Davis, M.D. (1997) Subretinal Fibrosis in Diabetic Macular Edema. ETDRS Report 23. Early Treatment Diabetic Retinopathy Study Research Group. Archives of Ophthalmology, 115, 873-877. http://dx.doi.org/10.1001/archopht.1997.01100160043006

[2] Begg, I.S. and Rootman, J. (1976) Clinico-Pathological Study of an Organized Plaque in Exudative Diabetic Maculopathy. Canadian Journal of Ophthalmology, 11, 197-202.

[3] Sigurdsson, R. and Begg, I.S. (1980) Organised Macular Plaques In Exudative Diabetic Maculopathy. British Journal of Ophthalmology, 64, 392-397. http://dx.doi.org/10.1136/bjo.64.6.392

[4] Dobree, J.H. (1970) Simple Diabetic Retinopathy. Evolution of the Lesions and Therapeutic Considerations. British Journal of Ophthalmology, 54, 1-10. http://dx.doi.org/10.1136/bjo.54.1.1

[5] Lewis, H., Schachat, A.P., Haimann, M.H., et al. (1990) Choroidal Neovascularization after Laser Photocoagulation for Diabetic Macular Edema. Ophthalmology, 97, 503-510, Discussion: 510-501.

[6] Berger, A.R. and Boniuk, I. (1989) Bilateral Subretinal Neovascularization after Focal Argon Laser Photocoagulation for Diabetic Macular Edema. American Journal of Ophthalmology, 108, 88-90. http://dx.doi.org/10.1016/S0002-9394(14)73271-4

[7] Han, D.P. Mieler, W.F. and Burton, T.C. (1992) Submacular Fibrosis after Photocoagulation for Diabetic Macular Edema. American Journal of Ophthalmology, 113, 513-521. http://dx.doi.org/10.1016/S0002-9394(14)74722-1

[8] Guyer, D.R., D’Amico, D.J. and Smith, C.W. (1992) Subretinal Fibrosis after Laser Photocoagulation for Diabetic Macular Edema. American Journal of Ophthalmology, 113, 652-656.

[9] Takagi, H., Otani, A., Kiryu, J. and Ogura, Y. (1999) New Surgical Approach for Removing Massive Foveal Hard Exudates in Diabetic Macular Edema. Ophthalmology, 106, 249-256, Discussion: 256-247.

[10] Ciardella, A.P., Klancnik, J., Schiff, W., Barile, G., Langton, K. and Chang, S. (2004) Intravitreal Triamcinolone for the Treatment of Refractory Diabetic Macular Oedema with Hard Exudates: An Optical Coherence Tomography Study. British Journal of Ophthalmology, 88, 1131-1136. http://dx.doi.org/10.1136/bjo.2004.041707 